Introdurre l'innovazione nella quotidianità. Riflessioni sul metodo e sugli strumenti utilizzati in una esperienza formativa in Cardiologia

\section{Effective implementation of change into routine work. Thinking over ways and means of a learning experience in Cardiology}

\section{Elisabetta Angelino}

\section{Servizio di Psicologia, Fondazione Maugeri, IRCCS, Torino.}

Corresponding author: Dott.ssa Elisabetta Angelino, Servizio di Psicologia, Fondazione Salvatore Maugeri, IRCCS; Presidio Ospedaliero Major via S. Giulia 60, I-10124 Torino; E-mail: elisabetta.angelino@fsm.it

ABSTRACT: Effective implementation of change into routine work. Thinking over ways and means of a learning experience in Cardiology. E. Angelino.

Effective implementation of change in patients' care is a substantive problem. Organizational learning is viewed as process of seeking, selecting, and adapting new "routines" to improve performance but learning from experience is not automatic, but rather may result from action and reflection within the organization.

Keywords: hospital discharge, intervention development, learning, checklist.

Monaldi Arch Chest Dis 2014; 82: 5-9.

\section{Introduzione}

L'attività lavorativa di ciascuno è quotidianamente attraversata e segnata da discussioni su ciò che è ritenuto opportuno fare o non fare, su ciò che è rappresentato come giusto, sbagliato, adeguato, inadeguato. Se al singolo è richiesto di saper fare una costante manutenzione e innovazione delle proprie competenze per offrire cure caratterizzate da qualità tecnica, relazionale e organizzativa, è necessario che l'attività formativa prenda davvero sul serio l'esigenza di partire dai problemi che le persone affrontano nei loro reali contesti lavorativi.

Implementare un intervento innovativo, seppure finalizzato a migliorare la qualità dell'assistenza con una maggiore efficacia e costo-efficacia delle cure, è sempre complicato e la modalità per raggiungere questo obiettivo non può essere quella classica della formazione frontale. L'impatto a lungo termine degli interventi innovativi dipende dalla possibilità di essere calati nel contesto reale dove viene richiesta, proprio dalle variabili di contesto, una flessibilità nella realizzazione. Secondo la "Normalisation process theory", ogni innovazione è per definizione un elemento di disturbo all'interno del contesto organizzativo, pertanto per introdurre interventi innovativi è opportuno non sovraccaricare il sistema di nuovi stimoli ma innescare il cambiamento, farlo consolidare nelle attività routinarie fino a "normalizzarlo", ovvero fino a che l'innovazione non rappresenta più un'innovazione. In questo senso è importante considerare anche l'impatto che l'intervento innovativo può avere sulla rete di relazione tra le diverse professionalità coinvolte nella sua realizzazione, quali siano le conseguenze che è in grado di apportare e quale il valore che le diverse professionalità attribuiscono a queste conseguenze [1].

Quanto premesso, indica come una formazione che possa migliorare la qualità delle cure, soprattutto quando necessita di introdurre nella routine quotidiana una innovazione, deve prendere spunto dai repertori reali di sapere, deve avvicinarsi ai processi organizzativi concreti e ai reali contesti di azione, deve essere sensibile alle loro sollecitazioni e fondata su una progettazione collaborativa e rispettosa dei ruoli e di tutti gli attori coinvolti [2].

\section{L'esperienza formativa}

Si propongono di seguito alcune riflessioni maturate durante una recente esperienza di formazione finalizzata alla diffusione di una modalità innovativa per il miglioramento della dimissione dopo Sindrome Coronarica Acuta.

L'obiettivo della logica formativa su cui è stata basata questa esperienza è stato quello di intercettare quanto costituiva il sapere pratico, costruito e distribuito localmente e poi di sottoporre questo sapere ad una elaborazione critica, confrontandolo con quanto evidenziato dalla letteratura. Considerando l'apprendimento un processo attivo, che avviene all'interno di una cornice partecipativa, e non una mera ricezione di informazioni, si è voluto così rinunciare alla tradizionale, ma illusoria, prospettiva di trasmissione di un sapere teorico/accademico che va a determinare un sapere professionale.

II progetto

II progetto formativo "Informazione alla dimissione dopo sindrome coronarica acuta", inserito nel Piano Formativo annuale ANMCO 2014, è stato rivolto, nel 2014, a medici e infermieri di strutture di Cardiologia per acuti di tre Regioni: Toscana, Campania, Liguria.

Il fine ultimo del progetto è il miglioramento della qualità della dimissione, attraverso l'adozione di una modalità condivisa e standardizzata della stessa che possa favorire, fin dal rientro a domicilio, l'intero percorso di cura del paziente. La letteratura sottolinea come la potenziale alta vul- 
nerabilità del paziente con esiti di evento coronarico renda cruciale il momento della dimissione in quanto la sua eventuale inadeguatezza finisce per ricadere sull'intera gestione del programma terapeutico [3].

\section{L'indagine teorica}

La metodologia adottata, è stata di tipo qualitativo e articolata in diversi momenti, distinti ma fortemente interconnessi, sulla base del principio secondo il quale ogni attività formativa dovrebbe essere in grado, non solo di migliorare le conoscenze dei partecipanti ma soprattutto di modificare i comportamenti professionali.

La prima fase del progetto, l'indagine teorica finalizzata all'analisi della letteratura recente, è stata propedeutica all'indagine sul campo, intesa come contestualizzazione nelle tre diverse regioni in cui si è deciso di sviluppare l'azione formativa.

L'attenzione è stata focalizzata sulla raccolta e sull'analisi di dati e modelli presentati dalla letteratura con la finalità di individuare le questioni importanti che consentissero:

- I'elaborazione di alcune prime ipotesi interpretative per mettere a fuoco i temi chiave da affrontare e su cui indirizzare la fase successiva;

- la costruzione degli strumenti da utilizzare.

Grazie all'indagine teorica sono emersi alcuni punti di attenzione su cui centrare l'azione formativa: "come fare" ovvero la gestione della dimissione con tutti gli aspetti organizzativi, rappresentati dal tempo necessario, dal luogo adeguato, dalla figura professionale, dal coinvolgimento del familiare, dalla verifica della comprensione delle informazioni; "cosa dire" ovvero i contenuti della dimissione con la scelta delle priorità, la selezione delle informazioni, la comunicazione del rischio, le istruzioni su farmaci, comportamenti, stile di vita; "come comunicare" ovvero la personalizzazione della dimissione con le diverse tipologie di pazienti, le risorse individuali su cui fare leva per migliorare l'aderenza, gli strumenti per favorirla, e la concreta fruibilità delle informazioni che vengono fornite.

\section{La ricerca sul campo}

La seconda fase del progetto, la ricerca sul campo, è stata condotta coinvolgendo medici e infermieri a cui, circa un mese prima dell'evento formativo, è stata inviata una scheda di rilevazione della modalità di dimissione per indagare la realtà operativa di ogni partecipante e per raccogliere aspettative e criticità sulla dimissione, un processo complesso e forse non così logico e lineare come viene descritto nella teoria (Tabella 1).

Il focus della ricerca sul campo è stato posto sui singoli professionisti e sul loro patrimonio di conoscenza con la finalità di evidenziare:

- quali competenze organizzative e individuali ci sono nelle diverse realtà

- dove è presente e dove invece manca una chiara definizione dei ruoli

\section{I risultati dell'indagine conoscitiva}

Esaminando le schede di rilevazione si è evidenziato un diverso giudizio formulato dai medici e dagli infermieri su alcune componenti della modalità di dimissione in atto nelle loro strutture. Nel valutare lo stile comunicativo, infatti, lo sguardo dei medici si è rivelato molto più critico soprattutto per quanto riguarda il riuscire ad identificare
Tabella 1

\begin{tabular}{|c|c|c|}
\hline A tuo giudizio l'abituale modalità di dimissione del paziente: & SI & NO \\
\hline Garantisce una adeguata continuità assistenziale & $\square$ & $\square$ \\
\hline $\begin{array}{l}\text { Garantisce una adeguata personalizzazione del percorso } \\
\text { di cura del singolo paziente }\end{array}$ & $\square$ & $\square$ \\
\hline $\begin{array}{l}\text { Tiene conto delle caratteristiche del singolo paziente } \\
\text { (es: età, livello di istruzione, condizione sociale) }\end{array}$ & $\square$ & $\square$ \\
\hline $\begin{array}{l}\text { Aiuta il medico e l'infermiere ad identificare le priorità } \\
\text { da comunicare al paziente }\end{array}$ & $\square$ & $\square$ \\
\hline Rende possibile coinvolgere adeguatamente i familiari & $\square$ & $\square$ \\
\hline $\begin{array}{l}\text { Ritieni che l'applicazione di una procedura standardizzata } \\
\text { di informazione potrebbe migliorare la qualità della dimissione }\end{array}$ & $\square$ & $\square$ \\
\hline Nella tua struttura per la dimissione del paziente: & SI & NO \\
\hline È disponibile un luogo idoneo & $\square$ & $\square$ \\
\hline L'organizzazione interna consente di dedicare un tempo adeguato & $\square$ & $\square$ \\
\hline $\begin{array}{l}\text { Vengono date informazioni sulla malattia e istruzioni su cosa fare } \\
\text { in presenza di sintomi }\end{array}$ & $\square$ & $\square$ \\
\hline Vengono date indicazioni verbali e scritte sui farmaci & $\square$ & $\square$ \\
\hline $\begin{array}{l}\text { Vengono date indicazioni sugli effetti dei farmaci ed i rischi } \\
\text { di interruzione }\end{array}$ & $\square$ & $\square$ \\
\hline $\begin{array}{l}\text { Vengono date informazioni sui fattori di rischio e sui comportamenti } \\
\text { di salute da adottare }\end{array}$ & $\square$ & $\square$ \\
\hline Viene verificata la comprensione del paziente & $\square$ & $\square$ \\
\hline Sei regolarmente coinvolto nelle attività sopraelencate & $\square$ & $\square$ \\
\hline Compilata da & & \\
\hline
\end{tabular}


le priorità da comunicare ed a personalizzare l'informazione. Interessante risulta il netto contrasto rispetto alla valutazione dell'avvenuta comprensione: la maggioranza dei medici ritiene che la comprensione non venga verificata mentre la maggioranza degli infermieri ritiene che la verifica venga effettuata regolarmente.

Le variabili organizzative, disponibilità di un luogo idoneo e possibilità di dedicare un tempo adeguato, appaiono invece essere percepite da medici e infermieri allo stesso modo e cioè come attualmente non adeguate.

\section{Dalle evidenze alle conoscenze}

Ritenendo che l'esperienza individuale e il ragionamento clinico, dei medici e degli infermieri, tutti "decisori esperti" nella modalità di dimissione, non possono essere sostituiti dalle evidenze della letteratura ma vadano ad esse integrati, si è cercato di approfondire e delineare i temi più sensibili tra quelli emersi dalla ricerca teorica, sollecitando la condivisione delle diverse esperienze sui problemi clinici percepiti come rilevanti.

Il quadro emerso dalla ricerca sul campo e dalla discussione in aula ha delineato uno scenario in forte evoluzione: i confini dei ruoli professionali per medici e infermieri risultano sempre meno definiti con la richiesta ad entrambi di avere competenze gestionali e organizzative. Gli infermieri appaiono essere il tessuto connettivo dell'ospedale, coloro che garantiscono la reale continuità assistenziale, ma tra loro appare essere molto diversificata la percezione della propria professionalità, le competenze possedute e l'approccio al lavoro. I cambiamenti strutturali all'interno di alcune organizzazioni ospedaliere (come per esempio il passaggio ad un modello per intensità di cura) rendono necessari cambiamenti anche nella modalità di relazione e di comunicazione con il paziente, ma all'evoluzione organizzativa spesso non corrisponde una evoluzione delle competenze relazionali con un conseguente più 0 meno esplicito disagio.

Nel contesto particolare di questa esperienza formativa, va sottolineato l'atteggiamento dei partecipanti che si sono mostrati desiderosi, rispetto ai temi trattati, di mettere in comune le questioni rilevanti perché si possano affrontare, elaborare e dunque gestire. La partecipazione attiva e le risposte spontanee hanno mostrato, nelle diverse strutture, l'esistenza di zone di ambiguità, di confusione dei ruoli e diversi livelli di evoluzione organizzativa. Nell'affrontare le tematiche relative alla modalità di dimissione, si è osservata una difficoltà da parte di molti a collocare gli atti di cura in una prospettiva temporale, cioè analizzando longitudinalmente la condizione del paziente, rispetto alle decisioni terapeutiche e quindi anche rispetto alle priorità da comunicare, per poter mantenere un atteggiamento equilibrato senza aggressività né abbandono.

E dato che "la continuità di cura deve essere un principio irrinunciabile di qualsiasi processo formativo" l'attenzione alla processualità è fondamentale [4].

\section{Dalle conoscenze ai comportamenti}

Nella fase di formazione in aula è stato proposta la check-list messa a punto per aiutare i medici e gli infermieri a strutturare il momento della dimissione, individuando le priorità da comunicare, personalizzando l'informazione sul singolo paziente, verificando la comprensione e controllando tempo e spazio dedicato (Tabella 2).

Si tratta di una check-list predisposta pensando non tanto ad una sua funzione pragmatica di "lista di controllo", ma piuttosto al suo potenziale essere un "filo di arianna" come aveva intuito Atul Gawande, colui che ha introdotto nel mondo sanitario le checklist proprio come strategie per governare le situazioni complesse e quindi "uscire dai labirinti" [5].

In questo senso la check-list non è un modulo da compilare - magari a posteriori - ed è qualcosa di più di un semplice strumento: essa rappresenta la sintesi delle evidenze e delle conoscenze che evocano la necessità di un comportamento e quindi il suo successo non dipende da quante caselle sono barrate ma da un modo di lavorare.

Con la convinzione che gran parte delle situazioni reali affrontate da medici e infermieri, alla dimissione, si presentano come aggrovigliate, caratterizzate da incertezza, disordine e indeterminatezza e dove il primo nodo da sciogliere è spesso definire il problema e stabilire le priorità, è stato chiesto esplicitamente ai partecipanti di utilizzare la check-list non con una rigida razionalità tecnica ma piuttosto come uno stimolo per riflettere nel corso dell'azione.

Quella che viene definita "riflessione nel corso dell'azione" [6] sta ad indicare come nella definizione dei problemi che si incontrano nella pratica professionale, fini e mezzi risultano interdipendenti, azione e riflessione si combinano tra di loro. Utilizzare la check-list in questo senso, vuol dire che ogni suo item rappresenta, per il medico e l'infermiere, uno stimolo per riflettere sulla situazione che si ha davanti e per definirla (formulando un'ipotesi); l'azione che segue e che verifica la definizione, rappresenterà l'introduzione di un cambiamento ma sarà anche occasione di auto-riflessione e apprendimento.

Per questo, la check-list non è stata messa a punto come un rigido strumento immodificabile e adatto a tutti i contesti, ma è stato chiesto ai partecipanti di sperimentarla nella propria realtà e poi eventualmente di proporre modifiche o adattamenti.

Infine l'importanza di una fase di feedback. La disponibilità e la capacità di dare feedback è, forse, ciò che più di tutto favorisce una reale evoluzione della relazione che si viene a creare con un progetto formativo, tra "chi insegna e chi apprende". Con un adeguato feedback si permette alla relazione di evolvere e quindi, alla fine del percorso, di essere, formatori e partecipanti, allo stesso livello e di condividere il contenuto e il significato dell'intervento innovativo su cui la formazione è stata condotta. Naturalmente un feedback adeguato non può essere il questionario ECM.

Dopo un periodo di sperimentazione della checklist è stato pertanto pianificato un feedback, con una valutazione delle diverse esperienze, una verifica di quanto fatto, delle osservazioni ricevute e delle even- 
Tabella 2

CheckList per dimissione dopo SCA

\begin{tabular}{lll}
\hline & $S i$ \\
\hline Disponibilità di un luogo idoneo & $\square$ \\
Definizione di un tempo dedicato & Se sì quanto previsto in media: minuti \\
\hline
\end{tabular}

\begin{tabular}{lll}
\hline \multicolumn{2}{c}{ Effettuato da } \\
Si & Medico Infermiere
\end{tabular}

Informazioni su malattia e prognosi

sulla malattia:

solo la diagnosi

spiegazioni sintetiche

spiegazioni in dettaglio

sulla prognosi:

nessuna indicazione

indicazioni specifiche

\begin{tabular}{ccc} 
Si & Medico & Infermiere \\
\hline & $\square$ & $\square$ \\
$\square$ & $\square$ & $\square$ \\
$\square$ & $\square$ & $\square$ \\
$\square$ & $\square$ & $\square$ \\
$\square$ & $\square$ & $\square$ \\
$\square$ & $\square$ & $\square$ \\
$\square$ & $\square$ & $\square$ \\
$\square$ & $\square$ & $\square$
\end{tabular}

Informazioni sui fattori di rischio

caratteristiche e significato F.di R. presenti come contenerli

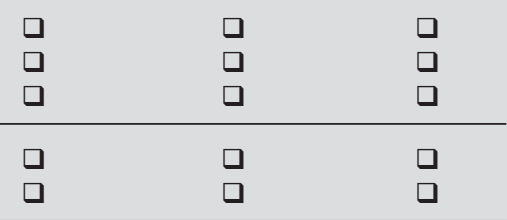

Informazioni sui sintomi

caratteristiche sintomi di allarme

$\begin{array}{lll}a & a & a \\ a & a & a \\ a & a & a \\ a & a & a\end{array}$

Istruzioni su uso dei farmaci

affidate a lettera di dimissione

fornite con spiegazioni verbali

consegna di schemi prestampati

\section{Informazioni su}

effetto dei farmaci

pericoli della interruzione

come ridurre il rischio di errori

\section{Informazioni sullo stile di vita}

dieta

attività fisica

lavoro

attività sessuale

guida dell'auto

Indicazioni per controlli e follow up

controlli da effettuare

controlli programmati

data e luogo degli stessi

\begin{tabular}{lll} 
a & a & a \\
a & a & a \\
a & a & a \\
\hline
\end{tabular}

data e luogo degli stessi

\begin{tabular}{lll}
$a$ & $a$ & $a$ \\
$a$ & $a$ & $a$ \\
$a$ & $a$ & $a$ \\
$\square$ & $a$ & $a$ \\
\hline
\end{tabular}

Le informazioni (contenuti e stile) sono state differenziate

per età

per sesso

per condizione culturale

per gravità della malattia

\begin{tabular}{lll}
$a$ & $a$ & $a$ \\
$a$ & $a$ & $a$ \\
$a$ & $a$ & $a$ \\
$a$ & $a$ & $a$ \\
$a$ & $a$ & $a$ \\
$a$ & $a$ & $a$ \\
\hline$a$ & $a$ & $a$ \\
$a$ & $a$ & $a$ \\
$a$ & $a$ & $a$ \\
$a$ & $a$ & $a$ \\
\hline
\end{tabular}

Consegna di materiale scritto/opuscoli

Verifica della avvenuta comprensione

domanda diretta (tutto chiaro?..)

è stato chiesto di ripetere le istruzioni ricevute

\begin{tabular}{|c|c|c|}
\hline $\begin{array}{l}\text { व } \\
\text { व } \\
\text { व } \\
\text { व }\end{array}$ & $\begin{array}{l}\square \\
\square \\
\square \\
\square \\
0\end{array}$ & $\begin{array}{l}\square \\
\square \\
\square \\
\square \\
\square\end{array}$ \\
\hline ם & a & 口 \\
\hline $\begin{array}{l}\text { व } \\
\text { a }\end{array}$ & $\begin{array}{l}\text { a } \\
\text { a }\end{array}$ & $\begin{array}{l}\text { a } \\
\text { a }\end{array}$ \\
\hline
\end{tabular}

Spazio alle domande del paziente

Pianificazione di un contatto telefonico per pazienti

a rischio di cattiva aderenza

Tempo totale dedicato (minuti):

Data di rilevazione: 
tuali proposte di modifica e adattamento. In questa fase si vuole anche valutare a che punto è il processo di costruzione del consenso nelle diverse strutture e se è stata avviata la discussione e la condivisione con i colleghi di quanto proposto dal progetto formativo.

Tutte le osservazioni raccolte e le proposte formulate, dopo la fase di sperimentazione, saranno poi elaborate e ulteriormente condivise tra tutti così da permettere, di pari passo con lo sviluppo di comportamenti professionali nuovi, anche una evoluzione e riformulazione della stessa check-list.

\section{Conclusioni}

L'aspetto innovativo del progetto è che, nonostante il proposito dichiarato di offrire uno "strumento" per indirizzare la pratica clinica (la checklist), sia stato evitato di elaborare e diffondere un rigido protocollo cui tutti dovevano attenersi. In un contesto in cui le linee-guida per la pratica clinica imperversano, si è scelto di optare, proprio nella fase formativa, per una dichiarata e aperta genericità di alcuni passaggi, in modo da concedere ai partecipanti un'interpretazione ampia delle diverse situazioni cliniche.

Non si è trattato di una scelta obbligata dalla mancanza di evidenze o di letteratura disponibile ma piuttosto di una scelta di campo, ponderata e consapevole. Con il superamento di una formazione un po'ingessata e basata solo sulle evidenze in favore di una formazione dinamica e fortemente basata sulle conoscenze, si è voluto permettere di esprimere valutazioni e di trovare soluzioni quanto più possibili inclusive e personalizzate sullo specifico contesto, sia a livello organizzativo che individuale.

\section{Riassunto}

Introdurre nella realtà clinica una pratica innovativa per migliorare la qualità delle cure rimane un problema rilevante. In genere si pensa che l'apprendimento organizzativo avvenga per tentativi ed errori con un progressivo adattamento alla nuova "routine": in realtà, all'interno del contesto organizzativo è l'agire riflessivo che consente l'apprendimento. Una formazione che possa migliorare la qualità delle cure deve essere in grado non solo di migliorare le conoscenze dei partecipanti ma soprattutto di modificare i comportamenti professionali. Vengono presentate alcune riflessioni derivate da un progetto formativo sul miglioramento della qualità della dimissione in Cardiologia, rivolto a medici ed infermieri.

Parole chiave: dimissione ospedaliera, formazione in cardiologia, check-list.

\section{Riferimenti Bibliografici}

1. Murray E et al. Normalisation process theory: a framework for developing, evaluating and implementing complex interventions. BMC 2010, 8: 63.

2. Alastra V, Kaneklin C, Scaratti G. La formazione situata. Repertori di pratica.2012. Edizioni Franco Angeli. Milano.

3. Fattirolli F, Angelino E. L'informazione essenziale e irrinunciabile alla dimissione dal reparto per acuti dopo Sindrome Coronarica - Parte 2: II progetto per migliorare la qualità delle cure. Monaldi Arch Chest Dis 2012; 78: 138-147.

4. Hirsh DA et al. "Continuity" as an Organizing Principles for Clinical Education Reform. N Engl J Med 2007; 335: 858-866.

5. Gawande A. The Checklist Manifesto: how to get things right. 2011. Picador. UK.

6. Schon D. II professionista riflessivo. Per una nuova epistemologia della pratica professionale. 1999. Edizioni Dedalo, Bari. 